
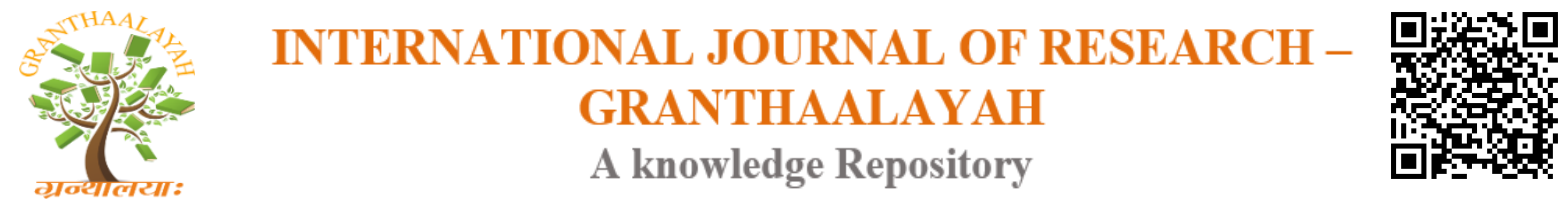

Science

\title{
AMELIORATED PARTICLE SWARM OPTIMIZATION ALGORITHM FOR SOLVING OPTIMAL REACTIVE POWER DISPATCH PROBLEM
}

\author{
Dr.K.Lenin *1 \\ ${ }^{* 1}$ Professor, Prasad V. Potluri Siddhartha Institute of Technology Kanuru, Vijayawada, Andhra \\ Pradesh -520007, India
}

\begin{abstract}
In this paper, an Ameliorated Particle Swarm Optimization (APSO) algorithm has been proposed to solve the optimal reactive power dispatch problem. Particle Swarm Optimization (PSO) is swarm intelligence-based exploration and optimization algorithm which is used to solve global optimization problems. But due to deficiency of population diversity and early convergence it is often stuck into local optima. Diversity upsurges and avoids premature convergence by using evolutionary operators in PSO. In this paper the intermingling crossover operator is used to upsurge the exploration capability of the swarm in the exploration space. Particle Swarm Optimization uses this crossover method to converge optimum solution in quick manner. Thus the intermingling crossover operator is united with particle swarm optimization to augment the performance and possess the diversity which guides the particles to the global optimum powerfully. Proposed Ameliorated Particle Swarm Optimization (APSO) algorithm has been tested in standard IEEE 30 bus test system and simulation results shows clearly the improved performance of the projected algorithm in reducing the real power loss and static voltage stability margin has been enhanced.
\end{abstract}

Keywords: Optimal Reactive Power; Transmission Loss; Intermingling Crossover Operator.

Cite This Article: Dr.K. Lenin. (2018). "AMELIORATED PARTICLE SWARM OPTIMIZATION ALGORITHM FOR SOLVING OPTIMAL REACTIVE POWER DISPATCH PROBLEM." International Journal of Research - Granthaalayah, 6(2), 202-213. https://doi.org/10.5281/zenodo.1189305.

\section{Introduction}

Reactive power optimization plays a key role in optimal operation of power systems. Many numerical methods [1-7] have been applied to solve the optimal reactive power dispatch problem. The problem of voltage stability plays a strategic role in power system planning and operation [8]. So many Evolutionary algorithms have been already proposed to solve the reactive power flow problem [9-11]. In [12, 13], Hybrid differential evolution algorithm and Biogeography Based algorithm has been projected to solve the reactive power dispatch problem. In $[14,15]$, a fuzzy based technique and improved evolutionary programming has been applied 
to solve the optimal reactive power dispatch problem. In $[16,17]$ nonlinear interior point method and pattern-based algorithm has been used to solve the reactive power problem. In [18-20], various types of probabilistic algorithms utilized to solve optimal reactive power problem. In this paper, an Ameliorated Particle Swarm Optimization (APSO) algorithm has been proposed to solve the optimal reactive power dispatch problem. Particle Swarm Optimization (PSO) [21] has been used efficaciously in solving many optimization problems, for its simplicity and fast convergence rate. Swarm intelligence is the subdivision of artificial intelligence and based on collective behaviour of self-organized system [22, 23]. The optimize value of the function using Particle Swarm Optimization Algorithm is hang on in the exploration and exploitation of the particles during searching in the exploration space [24].There are also problem in PSO like when it applies to various global optimization problems it may get held in the local optimization due to early convergence because the diversity shrinkages with the time for a large population[25], So we apply various evolutionary operator to get the global optimal solution[26-31].The intermingling crossover is a crossover operator which is applied in basic PSO to discover the exploration area . The intermingling crossover operator is a improved crossover operator, which is apply to the PSO to optimize the function. The proposed Ameliorated Particle Swarm Optimization (APSO) algorithm has been evaluated in standard IEEE 30 bus test system. The simulation results show that our proposed methodology outperforms all the entitled reported algorithms in minimization of real power loss.

\section{Voltage Stability Evaluation}

\subsection{Modal Analysis for Voltage Stability Evaluation}

Modal analysis is one among best methods for voltage stability enhancement in power systems. The steady state system power flow equations are given by.

$\left[\begin{array}{l}\Delta \mathrm{P} \\ \Delta \mathrm{Q}\end{array}\right]=\left[\begin{array}{cc}\mathrm{J}_{\mathrm{p} \theta} & \mathrm{J}_{\mathrm{pv}} \\ \mathrm{J}_{\mathrm{q} \theta} & \mathrm{J}_{\mathrm{QV}}\end{array}\right]\left[\begin{array}{l}\Delta \theta \\ \Delta V\end{array}\right]$

Where

$\Delta \mathrm{P}=$ Incremental change in bus real power.

$\Delta \mathrm{Q}=$ Incremental change in bus reactive Power injection

$\Delta \theta=$ incremental change in bus voltage angle.

$\Delta \mathrm{V}=$ Incremental change in bus voltage Magnitude

Jp $\theta$, JPV , JQ $\theta$, JQV jacobian matrix are the sub-matrixes of the System voltage stability is affected by both $\mathrm{P}$ and $\mathrm{Q}$.

To reduce (1), let $\Delta \mathrm{P}=0$, then.

$\Delta \mathrm{Q}=\left[\mathrm{J}_{\mathrm{QV}}-\mathrm{J}_{\mathrm{Q} \theta} \mathrm{J}_{\mathrm{P} \theta^{-1}} \mathrm{~J}_{\mathrm{PV}}\right] \Delta \mathrm{V}=\mathrm{J}_{\mathrm{R}} \Delta \mathrm{V}$

$\Delta \mathrm{V}=\mathrm{J}^{-1}-\Delta \mathrm{Q}$

Where 
$\mathrm{J}_{\mathrm{R}}=\left(\mathrm{J}_{\mathrm{QV}}-\mathrm{J}_{\mathrm{Q} \theta} \mathrm{J}_{\mathrm{P \theta ^{-1 }}} \mathrm{JPV}\right)$

$\mathrm{J}_{\mathrm{R}}$ is called the reduced Jacobian matrix of the system.

\subsection{Modes of Voltage Instability}

Voltage Stability characteristics of the system have been identified by computing the Eigen values and Eigen vectors.

Let

$\mathrm{J}_{\mathrm{R}}=\xi \wedge \eta$

Where,

$\xi=$ right eigenvector matrix of JR

$\eta=$ left eigenvector matrix of JR

$\Lambda=$ diagonal eigenvalue matrix of JR and

$\mathrm{J}_{\mathrm{R}^{-1}}=\xi \wedge^{-1} \eta$

From (5) and (8), we have

$\Delta \mathrm{V}=\xi \wedge^{-1} \eta \Delta \mathrm{Q}$

Or

$\Delta \mathrm{V}=\sum_{\mathrm{I}} \frac{\xi_{\mathrm{i}} \eta_{\mathrm{i}}}{\lambda_{\mathrm{i}}} \Delta \mathrm{Q}$

Where $\xi \mathrm{i}$ is the ith column right eigenvector and $\eta$ the ith row left eigenvector of JR. $\lambda i$ is the ith Eigen value of JR.

The ith modal reactive power variation is,

$\Delta \mathrm{Q}_{\mathrm{mi}}=\mathrm{K}_{\mathrm{i}} \xi_{\mathrm{i}}$

where,

$\mathrm{K}_{\mathrm{i}}=\sum_{\mathrm{j}} \xi_{\mathrm{ij}}{ }^{2}-1$

Where

$\xi \mathrm{ji}$ is the jth element of $\xi \mathrm{i}$

The corresponding ith modal voltage variation is

$\Delta \mathrm{V}_{\mathrm{mi}}=\left[1 / \lambda_{\mathrm{i}}\right] \Delta \mathrm{Q}_{\mathrm{mi}}$

If $|\lambda \mathrm{i}|=0$ then the ith modal voltage will collapse. 
In (10), let $\Delta \mathrm{Q}=\mathrm{ek}$ where ek has all its elements zero except the kth one being 1 . Then,

$\Delta \mathrm{V}=\sum_{\mathrm{i}} \frac{\eta_{1 \mathrm{k}} \xi_{1}}{\lambda_{1}}$

$\eta_{1 \mathrm{k}} \mathrm{k}$ th element of $\eta_{1}$

$\mathrm{V}-\mathrm{Q}$ sensitivity at bus $\mathrm{k}$

$\frac{\partial \mathrm{V}_{\mathrm{K}}}{\partial \mathrm{Q}_{\mathrm{K}}}=\sum_{\mathrm{i}} \frac{\eta_{1 \mathrm{k}} \xi_{1}}{\lambda_{1}}=\sum_{\mathrm{i}} \frac{\mathrm{P}_{\mathrm{ki}}}{\lambda_{1}}$

\section{Problem Formulation}

The objectives of the reactive power dispatch problem is to minimize the system real power loss and maximize the static voltage stability margins (SVSM).

\subsection{Minimization of Real Power Loss}

Minimization of the real power loss (Ploss) in transmission lines is mathematically stated as follows.

$P_{\text {loss }}=\sum_{k=(i, j)}^{n} g_{k}\left(V_{i}^{2}+V_{j}^{2}-2 V_{i} V_{j} \cos \theta_{i j}\right)$

Where $\mathrm{n}$ is the number of transmission lines, gk is the conductance of branch $\mathrm{k}, \mathrm{Vi}$ and $\mathrm{Vj}$ are voltage magnitude at bus $\mathrm{i}$ and bus $\mathrm{j}$, and $\theta \mathrm{ij}$ is the voltage angle difference between bus $\mathrm{i}$ and bus j.

\subsection{Minimization of Voltage Deviation}

Minimization of the voltage deviation magnitudes (VD) at load buses is mathematically stated as follows.

Minimize $V D=\sum_{\mathrm{k}=1}^{\mathrm{nl}}\left|\mathrm{V}_{\mathrm{k}}-1.0\right|$

Where $\mathrm{nl}$ is the number of load busses and Vk is the voltage magnitude at bus $\mathrm{k}$.

\subsection{System Constraints}

Objective functions are subjected to these constraints shown below.

Load flow equality constraints:

$P_{G i}-P_{D i}-V_{i \sum_{j=1}^{n b} V_{j}}\left[\begin{array}{cc}G_{i j} & \cos \theta_{i j} \\ +B_{i j} & \sin \theta_{i j}\end{array}\right]=0, i=1,2 \ldots, n b$ 
$Q_{G i}-Q_{D i}-V_{i} \sum_{j=1}^{n b} V_{j}\left[\begin{array}{cc}G_{i j} & \sin \theta_{i j} \\ +B_{i j} & \cos \theta_{i j}\end{array}\right]=0, i=1,2 \ldots, n b$

where, $\mathrm{nb}$ is the number of buses, $\mathrm{PG}$ and $\mathrm{QG}$ are the real and reactive power of the generator, PD and QD are the real and reactive load of the generator, and Gij and Bij are the mutual conductance and susceptance between bus $i$ and bus $j$.

Generator bus voltage (VGi) inequality constraint:

$\mathrm{V}_{\mathrm{Gi}}^{\min } \leq \mathrm{V}_{\mathrm{Gi}} \leq \mathrm{V}_{\mathrm{Gi}}^{\max }, \mathrm{i} \in \mathrm{ng}$

Load bus voltage (VLi) inequality constraint:

$\mathrm{V}_{\mathrm{Li}}^{\min } \leq \mathrm{V}_{\mathrm{Li}} \leq \mathrm{V}_{\mathrm{Li}}^{\max }, \mathrm{i} \in \mathrm{nl}$

Switchable reactive power compensations (QCi) inequality constraint:

$\mathrm{Q}_{\mathrm{Ci}}^{\min } \leq \mathrm{Q}_{\mathrm{Ci}} \leq \mathrm{Q}_{\mathrm{Ci}}^{\max }, \mathrm{i} \in \mathrm{nc}$

Reactive power generation (QGi) inequality constraint:

$\mathrm{Q}_{\mathrm{Gi}}^{\min } \leq \mathrm{Q}_{\mathrm{Gi}} \leq \mathrm{Q}_{\mathrm{Gi}}^{\max }, \mathrm{i} \in \mathrm{ng}$

Transformers tap setting (Ti) inequality constraint:

$\mathrm{T}_{\mathrm{i}}^{\min } \leq \mathrm{T}_{\mathrm{i}} \leq \mathrm{T}_{\mathrm{i}}^{\max }, \mathrm{i} \in \mathrm{nt}$

Transmission line flow (SLi) inequality constraint:

$S_{\mathrm{Li}}^{\min } \leq \mathrm{S}_{\mathrm{Li}}^{\max }, \mathrm{i} \in \mathrm{nl}$

Where, nc, ng and nt are numbers of the switchable reactive power sources, generators and transformers.

\section{Particle Swarm Optimization (PSO)}

PSO is a population-based optimization tool, where the system is initialized with a population of random particles and the algorithm searches for optima by updating generations. Suppose that the search space is $D$-dimensional. The position of the $i$-th particle can be represented by a $D$ dimensional vector $X_{i}=\left(x_{i 1}, x_{i 2}, \ldots, x_{i D}\right)$ and the velocity of this particle is $V_{i}=\left(v_{i 1}, v_{i 2}, \ldots, v_{i D}\right)$. The best previously visited position of the $i$-th particle is represented by $P_{i}=\left(p_{i 1}, p_{i 2}, \ldots, p_{i D}\right)$ and the global best position of the swarm found so far is denoted by $P_{g}=$ $\left(p_{g 1}, p_{g 2}, \ldots, p_{g D}\right)$. The fitness of each particle can be evaluated through putting its position into a designated objective function. The particle's velocity and its new position are updated as follows: 
$v_{i d}^{t+1}=\omega^{t} v_{i d}^{t}+c_{1} r_{1}^{t}\left(p_{i d}^{t}-x_{i d}^{t}\right)+c_{2} r_{2}^{t}\left(p_{g d}^{t}-x_{i d}^{t}\right)$

$x_{i d}^{t+1}=x_{i d}^{t}+v_{i d}^{t+1}$

Where $d \in\{1,2, \ldots, D\}, i \in\{1,2, \ldots, N\} N$ is the population size, the superscript $t$ denotes the iteration number, $\omega$ is the inertia weight, $r_{1}$ and $r_{2}$ are two random values in the range [0,1], $c_{1}$ and $c_{2}$ are the cognitive and social scaling parameters which are positive constants.

These both equations are used to update the velocity and position of a particle in the exploration space. The equation (24) is used to balance the search abilities of the particle in the search space. The equation (25) uses the velocity obtained in first equation to get the new position of the particle.

Crossover is a Genetic operator which is used after selection in Genetic Algorithm to get the new children using two or more than two parent. It is used to get the healthier solution than current solution. There are various improved version of crossover available to get the value of newfangled species. Intermingling crossover is also a improved operator which is used to get the new healthier child by using current parent. This operator is applied in PSO to optimize the multidimensional function and upsurge the probing capability of the PSO, So that Particle Swarm Optimization optimizes the functions efficiently and did not jammed in the local optima.

\section{Proposed Ameliorated Particle Swarm Optimization (APSO) algorithm}

Although the crossover operator is a conception of Genetic Algorithm but apart from genetic algorithm it has been used in many algorithms with some alterations. The crossover operator takes two or more than two parent and produce one or more than one child. The produced new child after crossover is superior to their parents. There are various improved crossover technique, The intermingling crossover operator is one of the improved crossover operator in which two particles are used to create a minimum and maximum range values which lies in the function's bounded region and the new particle is produced within the calculated minimum and maximum range values, Then we compute the fitness value of that new particle and compare it with the current particle and modernize the N_POP of the population of the particles.

\subsection{Intermingling Crossover}

Start

Select two arbitrary particles from N_POP $\mathrm{x}_{1}$ and $\mathrm{x}_{2}$

Compute $\mathrm{x}_{\text {new }}=\left(\mathrm{x}_{1}-\mathrm{x}_{2}\right)$

Compute $\mathrm{k}_{1}=\min \left(\mathrm{x}_{1}, \mathrm{x}_{2}\right)$

Compute $\mathrm{k}_{2}=\max \left(\mathrm{x}_{1}, \mathrm{x}_{2}\right)$

$\mathrm{k}_{\min }=\mathrm{k}_{1}-\mathrm{b}^{*} \mathrm{x}_{\text {new }}$;

$\mathrm{k}_{\max }=\mathrm{k}_{2}+\mathrm{b}^{*} \mathrm{x}_{\text {new }}$;

Where " $b$ " is an arbitrary selected integer within range

Now select an arbitrary particle from the range

N_new $=\left(\mathrm{k}_{\max }-\mathrm{k}_{\min }\right) *$ rand $+\mathrm{k}_{\min }$

Now compute the fitness of newly produced particle N_new 
End

\subsection{APSO Algorithm for Solving Optimal Reactive Power Dispatch Problem}

Start

Initialize particle with Arbitrary Position and Velocity

Set $\mathrm{P} \_$best $\mathrm{i}_{\mathrm{i}}=\mathrm{X}_{\mathrm{i}}, \mathrm{g} \_$best $=\min \left(\mathrm{P} \_\right.$best $\left.\mathrm{i}_{\mathrm{i}}\right)$

Initialize Generation as $\mathrm{g}=0$; While $\left(g<\max _{\text {generation }}\right)$

For ( $i=1$ to N_POP)

For $(j=1$ to D_POP)

Compute $v_{i d}^{t+1}$ using equation (11)

Compute $x_{i d}^{t+1}$ using equation (12)

If $v_{i d}^{t+1}$ and $x_{i d}^{t+1}$ are in exploration range then;

Calculate fitness for corresponding particle $\mathrm{x}_{\mathrm{i}}$;

Apply intermingling crossover to compute the new particle N_new

Compute fitness value for newlyproduced particle

Compare the fitness value for $\mathrm{x}_{\mathrm{i}}$ and $\mathrm{N} \_$new;

If Fitness ( $N$ new) is superior than $\mathrm{x}_{\mathrm{i}}$ then

Modernize the particle in N_POP $g=g+1$;

End for

End for

End of while

Print the value of $g \_$best.

End

\section{Simulation Results}

The efficiency of the proposed Ameliorated Particle Swarm Optimization (APSO) algorithm is demonstrated by testing it on standard IEEE-30 bus system. The IEEE-30 bus system has 6 generator buses, 24 load buses and 41 transmission lines of which four branches are (6-9), (6-10) , (4-12) and (28-27) - are with the tap setting transformers. The lower voltage magnitude limits at all buses are 0.95 p.u. and the upper limits are 1.1 for all the PV buses and 1.05 p.u. for all the PQ buses and the reference bus. The simulation results have been presented in Tables 1, 2, $3 \& 4$. And in the Table 5 shows the proposed algorithm powerfully reduces the real power losses when compared to other given algorithms. The optimal values of the control variables along with the minimum loss obtained are given in Table 1. Corresponding to this control variable setting, it was found that there are no limit violations in any of the state variables.

Table 1: Results of APSO - ORPD optimal control variables

\begin{tabular}{|l|l|}
\hline Control variables & Variable setting \\
\hline V1 & 1.040 \\
V2 & 1.041 \\
V5 & 1.045 \\
V8 & 1.030 \\
V11 & 1.000 \\
V13 & 1.030 \\
\hline
\end{tabular}




\begin{tabular}{|l|l|}
\hline T11 & 1.00 \\
T12 & 1.00 \\
T15 & 1.00 \\
T36 & 1.00 \\
Qc10 & 2 \\
Qc12 & 2 \\
Qc15 & 2 \\
Qc17 & 0 \\
Qc20 & 2 \\
Qc23 & 3 \\
Qc24 & 3 \\
Qc29 & 2 \\
Real power loss & 4.1268 \\
SVSM & 0.2472 \\
\hline
\end{tabular}

Optimal Reactive Power Dispatch problem (ORPD) together with voltage stability constraint problem was handled in this case as a multi-objective optimization problem where both power loss and maximum voltage stability margin of the system were optimized simultaneously. Table 2 indicates the optimal values of these control variables. Also it is found that there are no limit violations of the state variables. It indicates the voltage stability index has increased from 0.2472 to 0.2486 , an advance in the system voltage stability. To determine the voltage security of the system, contingency analysis was conducted using the control variable setting obtained in case 1 and case 2. The Eigen values equivalents to the four critical contingencies are given in Table 3. From this result it is observed that the Eigen value has been improved considerably for all contingencies in the second case.

Table 2: Results of APSO-Voltage Stability Control Reactive Power Dispatch Optimal Control Variables

\begin{tabular}{|l|l|}
\hline Control Variables & Variable Setting \\
\hline V1 & 1.044 \\
V2 & 1.046 \\
V5 & 1.043 \\
V8 & 1.031 \\
V11 & 1.000 \\
V13 & 1.031 \\
T11 & 0.090 \\
T12 & 0.090 \\
T15 & 0.090 \\
T36 & 0.090 \\
Qc10 & 3 \\
Qc12 & 3 \\
Qc15 & 2 \\
Qc17 & 3 \\
Qc20 & 0 \\
Qc23 & 2 \\
Qc24 & 2 \\
\hline
\end{tabular}




\begin{tabular}{|l|l|}
\hline Qc29 & 3 \\
Real power loss & 4.9882 \\
SVSM & 0.2486 \\
\hline
\end{tabular}

Table 3: Voltage Stability under Contingency State

\begin{tabular}{|l|l|l|l|}
\hline Sl.No & Contingency & $\begin{array}{l}\text { ORPD } \\
\text { Setting }\end{array}$ & $\begin{array}{l}\text { VSCRPD } \\
\text { Setting }\end{array}$ \\
\hline 1 & $28-27$ & 0.1419 & 0.1434 \\
\hline 2 & $4-12$ & 0.1642 & 0.1650 \\
\hline 3 & $1-3$ & 0.1761 & 0.1772 \\
\hline 4 & $2-4$ & 0.2022 & 0.2043 \\
\hline
\end{tabular}

Table 4: Limit Violation Checking Of State Variables

\begin{tabular}{|c|c|c|c|c|}
\hline \multirow[t]{2}{*}{ State variables } & \multicolumn{2}{|l|}{ Limits } & \multirow[t]{2}{*}{ ORPD } & \multirow[t]{2}{*}{ VSCRPD } \\
\hline & Lower & Upper & & \\
\hline Q1 & -20 & 152 & 1.3422 & -1.3269 \\
\hline Q2 & -20 & 61 & 8.9900 & 9.8232 \\
\hline Q5 & -15 & 49.92 & 25.920 & 26.001 \\
\hline Q8 & -10 & 63.52 & 38.8200 & 40.802 \\
\hline Q11 & -15 & 42 & 2.9300 & 5.002 \\
\hline Q13 & -15 & 48 & 8.1025 & 6.033 \\
\hline V3 & 0.95 & 1.05 & 1.0372 & 1.0392 \\
\hline $\mathrm{V} 4$ & 0.95 & 1.05 & 1.0307 & 1.0328 \\
\hline V6 & 0.95 & 1.05 & 1.0282 & 1.0298 \\
\hline V7 & 0.95 & 1.05 & 1.0101 & 1.0152 \\
\hline V9 & 0.95 & 1.05 & 1.0462 & 1.0412 \\
\hline V10 & 0.95 & 1.05 & 1.0482 & 1.0498 \\
\hline V12 & 0.95 & 1.05 & 1.0400 & 1.0466 \\
\hline V14 & 0.95 & 1.05 & 1.0474 & 1.0443 \\
\hline V15 & 0.95 & 1.05 & 1.0457 & 1.0413 \\
\hline V16 & 0.95 & 1.05 & 1.0426 & 1.0405 \\
\hline V17 & 0.95 & 1.05 & 1.0382 & 1.0396 \\
\hline V18 & 0.95 & 1.05 & 1.0392 & 1.0400 \\
\hline V19 & 0.95 & 1.05 & 1.0381 & 1.0394 \\
\hline V20 & 0.95 & 1.05 & 1.0112 & 1.0194 \\
\hline V21 & 0.95 & 1.05 & 1.0435 & 1.0243 \\
\hline V22 & 0.95 & 1.05 & 1.0448 & 1.0396 \\
\hline V23 & 0.95 & 1.05 & 1.0472 & 1.0372 \\
\hline V24 & 0.95 & 1.05 & 1.0484 & 1.0372 \\
\hline V25 & 0.95 & 1.05 & 1.0142 & 1.0192 \\
\hline V26 & 0.95 & 1.05 & 1.0494 & 1.0422 \\
\hline $\mathrm{V} 27$ & 0.95 & 1.05 & 1.0472 & 1.0452 \\
\hline V28 & 0.95 & 1.05 & 1.0243 & 1.0283 \\
\hline V29 & 0.95 & 1.05 & 1.0439 & 1.0419 \\
\hline V30 & 0.95 & 1.05 & 1.0418 & 1.0397 \\
\hline
\end{tabular}


Table 5: Comparison of Real Power Loss

\begin{tabular}{|l|l|}
\hline Method & Minimum loss \\
\hline Evolutionary programming [32] & 5.0159 \\
\hline Genetic algorithm [33] & 4.665 \\
\hline Real coded GA with Lindex as SVSM [34] & 4.568 \\
\hline Real coded genetic algorithm [35] & 4.5015 \\
\hline Proposed APSO method & 4.1268 \\
\hline
\end{tabular}

\section{Conclusion}

Ameliorated Particle Swarm Optimization (APSO) algorithm has been successfully solved optimal reactive power dispatch problem. In this paper the intermingling crossover operator is used to upsurge the exploration capability of the swarm in the exploration space. Particle Swarm Optimization uses this crossover method to converge optimum solution in quick manner. Thus the intermingling crossover operator is united with particle swarm optimization to augment the performance and possess the diversity which guides the particles to the global optimum powerfully. Proposed Ameliorated Particle Swarm Optimization (APSO) algorithm has been tested in standard IEEE 30 bus test system and simulation results shows clearly the improved performance of the projected algorithm in reducing the real power loss and static voltage stability margin has been enhanced.

\section{References}

[1] O.Alsac, and B. Scott, "Optimal load flow with steady state security”, IEEE Transaction. PAS 1973, pp. 745-751.

[2] Lee K Y ,Paru Y M , Oritz J L -A united approach to optimal real and reactive power dispatch , IEEE Transactions on power Apparatus and systems 1985: PAS-104 : 1147-1153

[3] A.Monticelli , M .V.F Pereira and S. Granville, "Security constrained optimal power flow with post contingency corrective rescheduling", IEEE Transactions on Power Systems :PWRS-2, No. 1, pp.175-182.,1987.

[4] DeebN, Shahidehpur S.M, Linear reactive power optimization in a large power network using the decomposition approach. IEEE Transactions on power system 1990: 5(2) : 428-435

[5] E. Hobson ,'Network consrained reactive power control using linear programming, ' IEEE Transactions on power systems PAS -99 (4) ,pp 868=877, 1980

[6] K.Y Lee, Y.M Park, and J.L Oritz, "Fuel -cost optimization for both real and reactive power dispatches", IEE Proc; 131C, (3), pp.85-93.

[7] M.K. Mangoli, and K.Y. Lee, "Optimal real and reactive power control using linear programming", Electr.Power Syst.Res, Vol.26, pp.1-10,1993.

[8] C.A. Canizares, A.C.Z.de Souza and V.H. Quintana, "Comparison of performance indices for detection of proximity to voltage collapse," vol. 11. no.3, pp.1441-1450, Aug 1996.

[9] Berizzi.C.Bovo, M.Merlo, and M.Delfanti, (2012), "A GA approach to compare ORPF objective functions including secondary voltage regulation," Electric Power Systems Research, vol. 84, no. 1, pp. $187-194$.

[10] D. Devaraj, and B. Yeganarayana, "Genetic algorithm based optimal power flow for security enhancement", IEE proc-Generation.Transmission and. Distribution; 152, 6 November 2005.

[11] A. Berizzi, C. Bovo, M. Merlo, and M. Delfanti, "A GA approach to compare orpf objective functions including secondary voltage regulation," Electric Power Systems Research, vol. 84, no. 1, pp. $187-194,2012$. 
[12] C.-F. Yang, G. G. Lai, C.-H. Lee, C.-T. Su, and G. W. Chang, "Optimal setting of reactive compensation devices with an improved voltage stability index for voltage stability enhancement," International Journal of Electrical Power and Energy Systems, vol. 37, no. 1, pp. $50-57,2012$.

[13] P. Roy, S. Ghoshal, and S. Thakur, "Optimal var control for improvement sin voltage profiles and for real power loss minimization using biogeography based optimization," International Journal of Electrical Power and Energy Systems, vol. 43, no. 1, pp. 830 - 838, 2012.

[14] B. Venkatesh, G. Sadasivam, and M. Khan, "A new optimal reactive power scheduling method for loss minimization and voltage stability margin maximization using successive multi-objective fuzzy lp technique," IEEE Transactions on Power Systems, vol. 15, no. 2, pp. 844 -851, may 2000.

[15] W. Yan, S. Lu, and D. Yu, "A novel optimal reactive power dispatch method based on an improved hybrid evolutionary programming technique," IEEE Transactions on Power Systems, vol. 19, no. 2, pp. $913-918$, may 2004.

[16] W. Yan, F. Liu, C. Chung, and K. Wong, "A hybrid genetic algorithm interior point method for optimal reactive power flow," IEEE Transactions on Power Systems, vol. 21, no. 3, pp. 1163 1169, aug. 2006.

[17] J. Yu, W. Yan, W. Li, C. Chung, and K. Wong, "An unfixed piecewise optimal reactive powerflow model and its algorithm for ac-dc systems," IEEE Transactions on Power Systems, vol. 23, no. 1 , pp. $170-176$, feb.2008.

[18] F. Capitanescu, "Assessing reactive power reserves with respect to operating constraints and voltage stability," IEEE Transactions on Power Systems, vol. 26, no. 4, pp. 2224-2234, nov. 2011.

[19] Z. Hu, X. Wang, and G. Taylor, "Stochastic optimal reactive power dispatch: Formulation and solution method," International Journal of Electrical Power and Energy Systems, vol. 32, no. 6, pp. $615-621,2010$.

[20] A. Kargarian, M. Raoofat, and M. Mohammadi, "Probabilistic reactive power procurement in hybrid electricity markets with uncertain loads," Electric Power Systems Research, vol. 82, no. 1, pp. $68-80,2012$.

[21] Kennedy J, EberhartR. Particle swarm optimization. Proceedings of the IEEE international conference on neural networks (Perth, Australia), 1942-1948. Piscataway, NJ: IEEE Service Center; 1995.

[22] Beni, G., Wang, J. Swarm Intelligence in Cellular Robotic Systems, Proceed. NATO Advanced Workshop on Robots and Biological Systems, Tuscany, Italy, June 26-30 (1989).

[23] P.N.Suganathan, N.Hansen, J.J.Liang, K.Dev, Y.PChen, A.Auger, S.Tiwari, Problems Definition and Evolution Criteria for the CEC 2005 Special Session on Real-Parameter Optimization.

[24] Pant, Millie, RadhaThangaraj, and Ajith Abraham. "A new pso algorithm with crossover operator for global optimization problems." Innovations in Hybrid Intelligent Systems. Springer Berlin Heidelberg, 2007. 215-222.

[25] Pant, Millie, RadhaThangaraj, and V. P. Singh. "Particle swarm optimization with crossover operator and its engineering applications." IAENG International Journal of Computer Science 36.2 (2009): 112-121.

[26] Zhang, Jian. "Particle swarm optimization using crossover operator." Journal of Convergence Information Technology 7.4 (2012): 287-295.

[27] Yu-hui Shi and Russell Eberhart," A modified particle swarm optimizer," in proceedings of IEEE world Congress on Computation intelligence,pp.69-73,1998.

[28] Takahashi, Masato, and Hajime Kita. "A crossover operator using independent component analysis for real-coded genetic algorithms." Evolutionary Computation, 2001. Proceedings of the 2001 Congress on. Vol. 1. IEEE, 2001. 
[29] Zhi-Feng Hao, Zhi-Gang Wang, HanHuang, A Particle Swarm Optimization Algorithm With Crossover Operator Proceedings of the 6th international conference on Machine Learning and Cybernatics ,Hong Kong, 19-22 August 2007.

[30] Chen, Stephen. "Particle swarm optimization with pbestcrossover."Evolutionary Computation (CEC), 2012 IEEE Congress on. IEEE, 2012.

[31] Qinghai bai, China "Analysis of Particle Optimization Algorithm" computer and information science (CCSE), Vol. 3, No. 1, Februry 2010.

[32] Wu Q H, Ma J T. "Power system optimal reactive power dispatch using evolutionary programming", IEEE Transactions on power systems 1995; 10(3): 1243-1248 .

[33] S.Durairaj, D.Devaraj, P.S.Kannan, "Genetic algorithm applications to optimal reactive power dispatch with voltage stability enhancement”, IE(I) Journal-EL Vol 87,September 2006.

[34] D.Devaraj, "Improved genetic algorithm for multi - objective reactive power dispatch problem", European Transactions on electrical power 2007; 17: 569-581.

[35] P. Aruna Jeyanthy and Dr. D. Devaraj "Optimal Reactive Power Dispatch for Voltage Stability Enhancement Using Real Coded Genetic Algorithm”, International Journal of Computer and Electrical Engineering, Vol. 2, No. 4, August, 2010 1793-8163.

\footnotetext{
*Corresponding author.

E-mail address: gklenin@ gmail.com
} 